\title{
Über die Fähigkeit des Periostes Knorpel zu bilden.
}

\author{
Von \\ Prof. Josef Schaffer \\ in Wien. \\ Mit Tafel V. \\ Eingegangen am 13. April 1897.
}

Veranlassung zu nachfolgenden Bemerkungen giebt mir eine von H. KoLLER ${ }^{1}$ ) vor Kurzem veröffentlichte Untersuchung, welehe hauptsächlich angestellt worden war, um Anfschlüsse über die Herkunft einer knorpelhaltigen Geschwulst am Kronenfortsatz des Unterkiefers zu erhalten. Da der Verfasser den Kronenfortsatz, gestützt auf die Angaben von Brock und Kassowirz für einen rein bindegewebig vorgebildeten Knochen hielt, so war für ihn »der partiell chondromatöse Tumor an einer Stelle entstanden, von der bis jetzt wenigstens nicht nachgewiesen ist, dass sie in der Norm je einmal fruiher knorpelig gewesen sei .

Daraus ergab sich die weitere Frage, ob denn das Periost eines bindegewebig vorgebildeten Knochen die Fähigkeit besitzt, unter gewissen Bedingungen Knorpel zu bilden. Auf Grund seiner Beobachtungen beantwortet der Verf. diese Frage dahin, dass das Periost bindegewebig vorgebildeter Knochen die latente und unter normalen Bedingungen nie hervortretende Eigenschaft der Knorpelproduktion besitzt.

1) Ist das Periost bindegewebig vorgebildeter Knochen im Stande Knorpel zu bilden? Experimentelle Untersuchung über den Einfluss durch einen äußeren Eingriff gesetzter Bedingungen auf die Entstehung eines bestimmten, an der betreffenden Stelle neuen Gewebes auf Basis latent vorhandener Anlage. Aus dem Privatlaboratorium des Privatdoc. Dr. HanaU in Zürich. Archiv f. Entwickelungsmechanik. Bd. III. 1896. pag. 624-656. 
Diese »durch die Arbeit des Herrn Dr. Koller festgestellte Thatsache" wird durch A. HANAU ${ }^{1}$ ) auch sofort in ein besonderes Licht gerïckt, indem HaNAU mit Bezug anf die von Roux aufgestellte Theorie von der Abhängigkeit der Knorpelbildung von gewissen mechanischen äußeren Einwirkungen, hervorhebt, dass diese Theorie durch die Arbeit seines Schülers Koller eine weitere und, wie ihm seheint, kräftige Stütze erhalten hat, ja, dass der von Koller erbrachte Nachweis die Wirkung dieses mechanischen äußeren Einflusses noch weit reiner darstellt, als es durch die früheren Erfahrungsthatsachen geschah, da er losgelöst erscheint von dem embryonal histogenetischen.

Mit diesen »früheren Erfahrungsthatsachen" nun haben es die beiden Autoren nicht selir genau genommen und ich glaube kaum, dass es zur Beantwortung der von Kollen aufgestellten Frage einer eigenen, experimentellen Untersuchung bedurft hätte. Dies schmälert jedoch durchaus nicht den Werth der von KolLER festgestellten Thatsachen, dass nämlich der Callus der rein bindegewebig vorgebildeten Schädelknochen beim Kaninchen nie, derjenige des Unterkiefers und Jochbogens stets und der des Supraorbitalrandes gelegentlich knorpeliger Natur ist.

Für den Unterkiefer war es schon durch zahlreiche Untersuchungen festgestellt, dass im Verlaufe seiner Entwicklung das Periost des ursprünglich rein periostal entstehenden Knochen an versehiedenen Stellen normaler Weise Knorpel zu bilden beginnt.

KoLLER selbst ist während seiner Litteraturstudien auf die Angabe von Brock ${ }^{2}$ ) gestoßen, dass beim Schwein der Angulus des Unterkiefers knorpeligen Ursprungs ist und auf die von KassowrTz ${ }^{3}$, dass auch im Processus glenoidalis Knorpelgewebe auftritt; wenn er das letztere, auctore Hertwig »nach den hentigen Kenntnissen" nicht als echten Knorpel anerkennt, so bätte ihn ein Durchschnitt durch einen embryonalen Gelenkfortsatz eines Besseren belehren können.

1) Nachtrag zn der Arbeit des Herrn Dr. Koller: Über die Beziehungen der durch die Arbeit des Herrn Dr. Kourer festgestellten Thatsache der chondroproduktiven Fähigkeit des Periostes rein bindegewebig vorgebildeter Knochen zu der von W. Roux aufgestellten Theorie über die Ursachen der Lokalisation der Knorpel- und Knochenbildung im Skelet. Archiv f. Entwickelungsmechanik. Bd. III. 1896. pag. 657-659.

2) Über die Entwickelung des Unterkiefers der Süugethiere. Zeitschr. . wiss. Zool. Bd. 27. 1876.

3) Die normale Ossifikation ete. Wien 1881, pag. 74 . 
Damit wäre eigentlich die ganze Fragestellung KoLLER's erledigt gewesen; er hilft sich jedoch über diese Thatsachen hinweg, indem er in den angeführten Befunden der nachträglichen Knorpelbildung am hinteren Ende des Unterkiefers (BRock) und in dem analogen (allerdings von Anderen bezweifelten) Vorgange an den Enden der Clavicula (KAssowirz), also an rein bindegewebig angelegten Knochen noch keine Entscheidung seiner Frage sieht: "Diese Vorgänge könnten wohl noch so gedeutet werden, dass ein bindegewebig vorher entstandener und ein nachträglich nebenan aus anderer Matrix gebildeter, knorpelig angelegter Knochen mit einander verschmelzen. "

Fiir eine solche $»$ Deutung $*$ geben aber die Schilderungen der beiden angeführten Autoren nicht den geringsten Anhalt, indem BRocK ${ }^{1}$ ) für die Knorpelbildung am Unterkiefer ausdrücklich betont, dass der Knorpel von Anfang an vom Periost umschlossen ist, nur aus Zellen der inneren Periostschicht entsteht und mit den Osteoblasten des anstoßenden Knochen direkt zusammenhängt. Ebenso wenig kann die Behauptung von Kassowicz'), dass das Periost zur Gelenkverbindung mit dem Schläfenbein einen Knorpel bildet, anders gedeutet werden, als dass es sich um dasselbe Periost handelt, welches bisher Knochen erzeugt hat. Ich selbst habe in einer eingehenden Untersuchung über die Verknöcherungsvorgänge am Unterkiefer ${ }^{3}$ ) aus einander gesetzt, dass bei der Entwickelung des Gelenkfortsatzes fruhzeitig eine Änderung des Gewebetypus eintritt, indem die Bildungszellen, d. b. Osteoblasten, welche zuerst Knochen producirt haben, im engsten räumlichen Zusammenhange mit diesem ein Übergangsgewebe zu Knorpel und endlich hyalinen Knorpel bilden.

Damit sind jedoch die Beweise dafür, dass im Lanfe der normalen Knochenentwickelung das Periost Knorpel bilden kann, nicht erschöpft.

Kassowitz, dessen Arbeit dem Autor ja bekannt war, führt außer den bereits genannten Stellen, an denen »normale Knorpelbildung im Periost ${ }^{4}$ ) vorkommt, auch noch die Tuberositas radii, die Symphyse des Unterkiefers und die Spina scapulae an. Nach KöLLIKER ${ }^{5}$ ) gehört auch die Gelenkgrube des Schläfenbeins hierher.

1) 1. c. pag. 295 .

2) 1. c. pag. 75 .

3) Die Verknücherung des Unterkiefers and die Metaplasiefrage. Archiv f. mikr. Anatomie. Bd. 32.1888.

4) l. c. V. Kap. pag. 67 und Med. Centralbl. 1877. Nr. 5, pag. 65.

5) Haudbuch der Gewebelehre. 6. Aufl. 1889. pag. 339. 
Diese Beispiele dürften genügen, um zu zeigen, dass die chondroproduktive Fähigkeit des Periostes rein bindegewebig entstehender Knochen schon lange anßer Frage gestellt war.

An dieser Thatsache ändert der Umstand nichts, dass es sich dabei um embryonal-histogenetische Vorgänge handelt, denn es wird kaum Jemand behaupten wollen, dass das Knochen producirende Periost des Embryo von dem des jugendlichen Individuums wesentlich verschieden ist.

Immerhin scheint es mir von Interesse, hier einer Stelle Erwähnung zu thun, an der auch beim Erwachsenen die metaplastische Fähigkeit des Periostes Knorpel zu bilden, wenn auch in beschränktem Maße, unter normalen Bedingungen zu Tage tritt. Dies ist der Fall an den Endphalangen der Finger, vielleicht auch an denen der Zehen.

Das Nagelglied der Endphalangen ist bekanntlich ein rein bindegewebig entstehender Knochen (ScHÄFer, Dixey) ${ }^{1}$ ). An Durchschnitten durch dasselbe (Fig. 1) finde ich nun an der Oberflache des Knochens zwischen den einstrahlenden Sehnenenden, die hier theilweise gleichzeitig das Periost bilden $(P)$, neben typischen Osteoblasten Zellen, welche eine deutliche Kapsel besitzen, um welche sich nicht selten ein homogener Hof von Grundsubstanz entwickelt zeigt, der durch intensive Färbbarkeit mit Hämatoxylin ansgezeichnet ist (Fig. $1 / n$ ).

Die Höfe benachbarter Zellen können zusammenfließen, so dass dann blasige, mit Kapseln versehene Zellen in einer mit Hämatoxylin blau gefärbten Grundsubstanz eingeschlossen erscheinen, das typische Bild des hyalinen Knorpels. Dass diese Knorpelbildung ebenfalls nur eine provisorische ist, erkennt man an den Resorptionsbuchten, welche die dinne Knochenrinde der Phalange $(P K)$ zeigt, deren blau färbbare Säume als Kitt- und Ansatzlinien auch noch in den oberflächlichen Bälkchen der Spongiosa, ebenso wie in der Knochenrinde sichtbar sind.

Die hier geschilderte Knorpelbildung ist offenbar in Analogie zu setzen mit der, welche RAvvier ${ }^{2}$ ) und Rexact ${ }^{3}$ ) in Sehnen-

1) Schüfer, E., and Dixer. F.. Preliminary note of the ossification of the terminal phalanges of the digits. Proc. of the roy. soc. of London. Vol. XXX. No. 205, 1880. - Drxey, F., On the ossification of the terminal phalanges of the digits. Ibidem No. 207 .

2 Les éléments et les Tissus du système conjonctif. Jonrn. de Mikrographie. XIV. Année. No. 10. 1890 und Traité technique Deutsche Übersetzung. pag. 383 .

3. Traité d'Histologie pratique. T. I. Paris 1885. pag. 355. 
ansätzen an hyalinen Knorpel beschreiben. Dieselbe Erscheinung kann man aber auch an vielen anderen Stellen sehen, wo Sehnengewebe in periostalen Knochen einstrahlt. Untersucht man z. B. den Ansatz der Achillessehne an den Tuber calcanei vom Kalb (Fig. 2) an sagittalen Längsschnitten, so findet man die Sehnenbündel direkt in den Knochen einstrahlen.

Zwischen ihnen baben die reihenweise geordneten Zellen eine Metamorphose in typische Knorpelzellen erfahren, so dass unmittelbar an den Knochen, der ja auch rein periostal entstanden ist, ein echter Faser- oder Bindegewebsknorpel $(\boldsymbol{F} K)$ stößt, welcher ebenfalls wieder der Resorption verfällt, um dem Knochengewebe Platz zu machen $(R)$.

Das Periost, welehes hier die Knochenbälkchen des Tuber calcanei erzeugt, bildet andererseits auch echte Knorpelzellen, so dass zwischen dem Faserknorpel, dessen Grundsubstanz der Hauptsache nach aus Faserbiindeln besteht und dem periostalen Knochen $(P K)$ diunne Lagen echten Hyalinknorpels $(K p)$ eingeschaltet erscheinen, in denen Resorption und Einlagerung neoplastischen Knochens $\left(P K^{\prime}\right)$ stattfindet, wie bei der endochondralen Ossification.

Diese Knorpelzellen führe ich jedoch nicht, wie Raxrier ${ }^{1}$ ), auf eine Lmwandlung bereits typisch-differenzirter Sehnenzellen zurück, ron welchem Vorgange Ravvier eine sehr eigenthümliche Darstellung giebt. Die Knorpelzellen gehen hier vielmehr aus indifferenten Bildungszellen hervor, welche sich - sichtlich unter verschiedenen mechanischen Einflüssen - theils in Knochenzellen, theils in Knorpelzellen, theils endlich in typische Sehnenzellen umwandeln können.

Solche Änderungen des Gewebetypus sind dem Histologen längst bekannt: das Periost ist zuerst Perichondrium und kann dann zeitweilig wieder zu Perichondrium werden. Dieser Fall kann aber auch eintreten, ohne dass es je Perichondrium war, nur wird es danu wieder Periost. In der Gegend der sog. encoche kann man lange Zeit hindurch beobachten, dass das gleiche Bildungsgewebe im engsten räumlichen Zusammenhange Knochen und hyalinen Knorpel erzeugt. Das Chordaepithel kann nach den neuesten Untersuchungen von v. EBNER 2) eine Reihe morphologisch und mikrochemisch höchst

1 Journ. d. Micrographie. XV. Année. 1891. No. 7.

2) Die Chorda dorsalis der niederen Fische und die Entwickelung des Bindegewebes. Zeitschr. f. wiss. Zool. Bd. 62. 1896. pag. 469. 
verschiedener Gewebetypen erzengen, so lange es den Charakter indifferenter Bildungszellen beibehält.

Diese Thatsachen, welche sich leicht noch durch eine Reihe anderer Beispiele vermehren ließen, sind lange Zeit falseh gedeutet worden, indem man die räumlichen Übergänge dieser verschiedenen Gewebe gleichzeitig als genetische auffasste, $d . h$. aus einem fertigen Gewebe, z. B. dem hyalinen Knorpel oder der Chorda, Knochen oder Knorpel in der Weise hervorgehen ließ, dass sich die roll differenzirte Knorpelzelle in ein Knochenkörperchen, die blasige Chordazelle in eine Knorpelzelle umwandelin sollte.

Diese metaplastische Anschaung wird heute noch von vielen Autoren wenigstens für einige Objekte festgehalten, unter denen der Unterkiefer die größte Rolle spielt. Meine Untersuchungen an einer geschlossenen Entwickelungsreihe des Unterkiefers rom Schaf haben die Haltlosigkeit einer solehen metaplastischen Anschauung darzulegen versucht und an ihre Stelle die metaplasirende Fähigkeit des indifferenten Bildungsgewebes zur Erklärung räumlicher und genetischer Gewebeübergänge herangezogen.

Für den Unterkiefer sind meine Anschaungen durch die unten eitirte Arbeit von Henneberg bestätigt worden.

Die Ursachen für solche Änderungen im Gewebetypus mögen vielfach in mechanisch-funktionellen Verhältnissen begründet sein und glaube ich, dass die Erklärung derselben keine uninteressante Aufgabe für die Entwickelungsmechanik sein wird.

Dass die bisher vorliegenden Versuche von-Roux ${ }^{1}$ ), jegliche Knorpelbildung auf die mechanische Wirkung von Abscherung und Druck zurtickzuführen, nur eine theilweise Erklärung zu geben vermögen, hat HaNaU mit Recht hervorgehoben ${ }^{2}$ ).

Für die Knorpelbildung bei niederen Thieren müssen wir uns stets die Thatsache vor Augen halten, dass das Knorpelgewebe gegenüber dem Knochengewebe das phyletisch ältere 3 ) und histologisch einfachere, eines rascheren Wachsthums fähigere ist. Letzterer Umstand dürfte auch seine provisorische Verwendung bei der Entwickelung des Skelettes der höheren Thiere vielfach verständlich

1) Gesammelte Abhandlungen über Entwickelungsmechanik. Leipzig 1895. Bd. I. pag. 810 . Bd. II. pag. 328 .

2) Nach der Darstellung von Roux kïmen die mechanischen Momente von Abscherung und Druck allerdings hauptsïchlich nur für die Erhaltung and das spätere Knorpelwachsthum in Betracht.

3) Busch, Zur weiteren Begründung der Osteoblastentheorie. Verhandl. d. phys. Ges. zu Berlin. 1879. Nr. 10. 
machen und verweise ich betreffs dieses Punktes auf die von mir citirten und speciell zur Erklärung der Knorpelbildung am Unterkiefer herangezogenen Anschaunngen von H. Müluler, Lovév, StrelzofF etc., welche ich in den Satz zusammenfassen konnte1), dass der Knorpel dort, wo er durch metaplasirende Thätigkeit des Periostes entsteht, eine rein provisorische Bedeutung besitzt, gleichsam als Modell dient, um welches die Knochenform gegossen wird und welches alsbald wieder der Resorption anheimfällt, um dem definitiven Ausgusse mit Knochen Platz zu machen.

Durch die vorstehenden Ausführungen glaube ich gezeigt zu haben, dass die Thatsache der Knorpelbildung von Seiten des Periostes rein bindegewebig entstandener Knochen keine neue ist und nicht erst eines experimentellen Nachweises bedurfte.

Was nun den eigentlichen Ausgangspunkt der Arbeit KolLER's, eine Erklärung für das Vorkommen einer knorpeligen Geschwulst am Kronenfortsatz des Unterkiefers zu suchen, anlangt, so ist es nicht uninteressant, dass KoLLER selbst und zwar durch ziemlich weitläufige theoretische Betrachtnngen tiber die verschiedenen Ursachen der Callusbildung, sowie über die Prädisposition eines embryonal angelegten Knorpels im Gegensatze zu derjenigen des regenerativ entstandenen zur Enchondrombildung auf die Vermuthnng kommt, ob nicht auch am Kronenfortsatze im Laufe seiner Entwickelung Knorpel vorkomme.

Auf eine diesbezügliche Anfrage Hanau's wurde Kollen von Prof. G. Bons in Breslau auf die Arbeit von Masquelin ${ }^{2}$ ) aufmerksam gemacht, in welcher das Vorkommen eines Knorpelkernes im Kronenfortsatz des menschlichen Unterkiefers nachgewiesen wird.

In meiner oben citirten Abhandlung, die dem Verfasser entgangen ist, habe ich mich ebenfalls eingehender mit der Knorpelbildung im Kronenfortsatze beschäftigt und einerseits die Geschichte derselben zusammengestellt, aus welcher hervorgeht, dass vor MAsquelin bereits BRUCH $^{3}$ ) und StRELzoFF ${ }^{4}$ ) Knorpelgewebe oder Reste desselben im Processus coronoides beobachtet haben, andererseits nachweisen

1) 1. c. pag. 333 .

2) Recherches sur le développement da maxillaire inférieur. Bull. de "Acad. roy. de Belgique. 2. Sér. T. XLV. No. 4. 18i8. Koller eitirt angeblich uach Minot T. XIV. 18i4. Mrvot citirt richtig 1878 und XIV ist Druckfehler.

3) Denkschr. d. Schweiz. naturforsch. Ges. Bd. XII. 1843.

4) Untersuchungen aus d. pathol. Instit. zu Zürich. Herausgegeben von Eberth. 1873. H. 1. 
können, dass im Kronenfortsatze beim Schafembryo ein schmaler, langer Knorpelkern als relativ späte, aber direkte Bildung des ursprünglichen Periostes auftritt und dass derselbe seine Funktion noch während des Fötallebens vollendet, so dass gegen Ende desselben keine Spur von Knorpel mehr im Kronenfortsatz gefunden wird.

Diese meine Angaben wurden erst kürzlich von einem Schüler O. Hertwig's, B. Henneberg ${ }^{1}$ ), am Menschen nachuntersucht und im Wesentlichen bestätigt.

Henneberg beobachtete das Auftreten von Knorpel im Kronenfortsatz beim Embryo von $11 \mathrm{~cm}$ Scheitelsteißlänge - also ebenfalls relativ spät, da die Knorpelbildung im Gelenkfortsatz schon beim $6,5 \mathrm{~cm}$ langen und die in der Symphyse beim $7,5 \mathrm{~cm}$ langen Embryo im Gange ist - und scheint es ihm unzweifelhaft, dass der hier als Perichondrium auftretende Theil des Unterkieferperiostes als Matrix des Knorpels anzusehen ist. Bei Embryonen von $15 \mathrm{~cm}$ hat die Knorpelbildung bereits ihre Höhe erreicht und beim Embryo von $18 \mathrm{~cm}$ Länge sind im Kronenfortsatz bereits keine Spuren von Knorpel mehr zu entdecken.

Zum Schlusse sei noch erwähnt, dass auch bereits experimentelle Erfahrungen iiber die chondroproduktive Fähigkeit des Periostes vorliegen. Ich verweise, um nur ein Beispiel herauszugreifen, auf die Untersuchungen von RigaL und VignaL ${ }^{2}$, welche mir, obwohl sie an Röhrenknochen angestellt wurden, für die aufgeworfene Frage vollkommen beweiskräftig scheinen. Die mittleren Partien eines Röhrenknochen vom Erwachsenen sind als rein periostal gebildeter Knochen anzusehen. Der Callus, welcher an solchen Stellen bei komplicirten Frakturen bei Ausschluss jeglicher Eiterung auftrat, war knorpelig, so dass die Verfasser selbst zu dem Schlusse kamen, dass dem Periost allein die Fähigkeit Knorpel zu bilden zukomme.

Von besonderem Interesse scheinen mir noch die Experimente, welche $\mathrm{M}_{A A S^{3}}$ ) an den marklosen Oberarmknochen der Vögel - er wählte dazu möglichst alte Thiere - angestellt hat, weil man die

1) Beiträge zur Entwickelungsgeschichte des Unterkiefers beim Menschen. Diss. Berlin 1894.

2) Sur la formation du cal. Compt. rend. T. 90. No. 21. 1880. - Recherches expérimentales sur la formation $d u$ cal et sur les modifications des tissus dans les psendarthroses. Arch. de physiol. No. 3 a. 4.1881.

3) Über das Wachsthum und die Regeneration der Röhrenknochen mit besonderer Berücksichtigung der Callusbildung. Archiv für klin. Chirurgie. Bd. XX. H. 4. 
mittleren Partien dieser Knochen, so weit sie für die Versuche in Betracht kamen, sicher als rein periostal entstandene ansehen kann. Der bei Frakturirung dieser Stellen auftretende Callus stellte zu einer gewissen Zeit eine Knorpelmasse dar, welche kontinuirlich außen um die Bruchenden, zwischen ihnen und in der (marklosen) Markhöhle liegt.

Wenn ich in den vorliegenden Ausführungen nun auch keine wesentlichen, neuen Thatsachen beibringen konnte, so glaube ich doch die Absicht, die mich bei denselben geleitet hat, hinlänglich deutlich dargethan zu haben. In dem Maße, als die Zahl der wissenschaftlichen Arbeiten auf unserem Gebiete zunimmt, muss auch die Vorsicht bei der Feststellung »neuer Thatsachen « und die Gewissenhaftigkeit in der Benutzung älterer begründeter Angaben zunehmen; sonst werden derartige Arbeiten zu einem unnützen Ballast unserer Litteratur.

\section{Erklärung der Abbildungen.}

Tafel V.

Fig. 1. Ans einem Querschnitt durch die Endphalange (Nagelglied) eines Fingers vom Hingerichteten. $P$ Perichondrium, beziehungsweise Sehneninsertion. $K n$ Knorpelzellen, $P K$ periostale Knochenrinde, $O b$ Osteoblastenlager des Markes, OK Osteoklast, $S p$ Spongiosabälkchen, $F$ Fettzellen. Vergr. 180.

Fig. 2. Längssehnitt durch den Ansatz der Achillessehne an den Tuber calcanei vom Kalb. $P K$ periostaler Knochen, $F K$ in Faserknorpel umgewandelte Sehne, $K n z$ Knorpelzellen, $K p$ homogener Knorpel, $R$ Resorptionslïcken im Knorpel. $P K^{\prime}$ in dieselben eingelagerter Knochen. Vergr. 160. 